Bull. Austral. Math. Soc.

VOL. 56 (1997) [175-176]

\title{
Functional equations of iterative type
}

\author{
MARIUSZ BAJGER
}

We solve some functional equations (in several variables) in which compositions of unknown functions appear. Our main results concern the iterative Cauchy and Pexider equations, and some of their generalizations (Cauchy and Pexider differences, the Daróczy equation, the translation equation.) Some other (non-iterative) functional equations are occasionally studied.

Let $K$ be a groupoid and $D(K)$ be the domain of the binary operation on $K$. We consider the following generalized Pexider equations:

$$
F(s t)=k(s t) \circ H(s) \circ G(t), \quad(s, t) \in D(K),
$$

where the functions $F, G, H, k$ to be determined are defined on $K$ and take values in some function spaces (with compositions of functions as a binary operation). Treating $k$ as a given function, under the basic assumption that each element of $K$ has left and right units, we present the general solution of (1) by reducing it to the Cauchy equation

$$
T(s t)=T(s) \circ T(t), \quad(s, t) \in D(K) .
$$

Further, we generalize the notion of the Cauchy and Pexider differences for functions taking values in some function spaces (not necessarily invertible functions) providing solutions of the equation

$$
F(s t)=p(s, t) \circ H(s) \circ G(t), \quad(s, t) \in D(K),
$$

with respect to functions $F, G, H$ defined on $K,\left(p\right.$ is a given function defined on $\left.K^{2}\right)$, by reducing it to the corresponding generalized Cauchy equation.

Assuming that $K$ is a field, we study (1) for two binary operations, that is, in the form

$$
F(s+t)=k(s t) \circ H(s) \circ G(t), \quad s, t \in K
$$

Received 28th February, 1997

Thesis submitted to the University of Queensland, August 1996. Degree approved, December 1996. Supervisor: Dr JH Chabrowski.

Copyright Clearance Centre, Inc. Serial-fee code: 0004-9729/97 \$A2.00+0.00. 
and solve it with respect to four unknown functions $F, G, H$ and $k$ mapping $K$ into suitable function spaces.

Since any group can be considered as a group of transformations of a set, our results for equations (1), (3), (4) imply corresponding ones for functions $F, G, H, k, p$ taking values in an arbitrary abstract group.

Using iteration theory methods, we investigate families of functions $\{T(t): t \in \mathbb{Q}\}$ $(\mathbb{Q}$ stands for rational numbers) satisfying (2) (rational flows) mapping a real open interval $I$ into itself. It is shown that every non-dense rational flow of homeomorphisms on $I$ with no fixed points, is conjugate with a special rational flow of piecewise linear homeomorphisms on $I$ (which we construct). Since the corresponding result for dense rational flows is known, it provides a complete description of all such flows.

As an application of these techniques of iteration theory we obtain the general solution of the translation equation in a class of increasing functions.

Finally, using our results for equations (1), (2) jointly with some other facts from iteration theory, we solve (1) for functions $F, G, H, k$ defined on the additive group of reals (rationals) and taking values in some special function spaces.

43 B Brookvale Rd

Windsor Gardens SA 5087

Australia 